\title{
The Graph of Simplex Vertices
}

\author{
M. El-Ghoul \\ Mathematics Department, Faculty of Science, Tanta University, Tanta, Egypt \\ Tel: 104-684-812 E-mail: m_elghoul@hotmail.com \\ N. El-Obaidi \\ Mathematics Department, Faculty of Science, Girl's College, Cairo, Egypt
}

Tel: 164-306-771 E-mail: najat_alobaidi@yahoo.com

Received: June 27, 2011 Accepted: July 15, 2011 Published: February 1, 2012

doi:10.5539/jmr.v4n1p105 URL: http://dx.doi.org/10.5539/jmr.v4n1p105

\begin{abstract}
In this paper we will introduce a new types of graph. The representation of the new graph by adjacent and incidence matrices will be obtaind. Some geometric transformations on the new graphs are described.
\end{abstract}

Keywords: Graphs, Simplex

2000 Mathematics subject classification: 51H10, 57N10

\section{Definitions and Background}

(1) Abstract graph: An abstract graph $G$ is a diagram consisting of a finite non empty set of the elements, called "vertices" denoted by $V(G)$ together with a set of unordered pairs of these elements, called "edge" denoted by $E(G)$. The set of vertices of the graph $G$ is called "the vertex-set of $G$ " and the list of edges is called "the edge -list of $G$ " (Giblin, 1977; Gibbson, 1995).

(2) Simplex: Given any set $V=\left\{v_{0}, v_{1}, \ldots, v_{n}\right\}$ of $n+1$ points in $R^{n}$, such that the differences $v_{1}-v_{0}, v_{2}-v_{0}, \ldots, v_{n}-v_{0}$ are linearly independent, the n-simplex with vertices $V$ is the convex hull of $V$, i.e. the set of all points of the form $t_{0} v_{0}+t_{1} v_{1}+\ldots+t_{n} v_{n}$, where $\sum_{i=0}^{n} t_{i}=1$ and $t_{i} \geq 0$ for all $i$ (Hatcher, 2002).

(3) Adjacency and Incidence: let $v$ and $w$ be vertices of a graph if $v$ and $w$ are joined by an edge $e$. Then $v$ and $w$ are said to be adjacent, moreover, $v$ and $w$ are said to be incident with $e$, and $e$ is said to be incident with $v$ and $w$ (Wilson, 1972).

(4) The adjacency matrix: let $G$ be a graph without loops, with $n$-vertices labeled $1,2,3, \ldots, n$. The adjacency matrix $A(G)$ is the $n x n$ matrix in which the entry in row $i$ and column $j$ is the number of edges joining the vertices $i$ and $j$ (Wilson, 1972).

(5) The incidence matrix: let $G$ be a graph without loops, with $n$-vertices labeled $1,2,3, \ldots, n$ and $\mathrm{m}$ edges labeled $1,2,3, \ldots, m$. The incidence matrix $I(G)$ is the $n x n$ matrix in which the entry in row $i$ and column $j$ is 1 if vertex $i$ is incident with edge $j$ and 0 otherwise (Wilson \& Watkins, 1990; Gross \& Tucker, 1987).

(6) Folding and unfolding of graph:

(a) Let $f: G \longrightarrow \bar{G}$ be a map between any two graphs $G, \bar{G}$ and (not necessary to be simple) such that if (u,v) $\in$ $G,(f(u), f(v)) \in \bar{G}$. Then $f$ is called a "topological folding" of $G$ to provided that $d(f(u), f(v)) \leq d(u, v)($ Giblin, 1977).

(b) Let $g: G \longrightarrow \bar{G}$ be a map between any two graphs $G, \bar{G}$ and (not necessary to be simple) such that if (u,v) $\in$ $G,(g(u), g(v)) \in \bar{G}$. Then $g$ is called a "topological unfolding" of $G$ to provided that $d(g(u), g(v))>d(u, v)($ El-Ghoul, 2007).

\section{Main Result}

Now we will define and discuss the graph of simplex vertices and some transformations on this new graph, the incident and adjacent matrices which represent these new graphs will be discussed.

\subsection{Definition}

The graph of simplex vertices is a pair $(V(G), E(G))$ where $V(G)=\left\{\left\{V_{0}\right\},\left\{V_{1}\right\}, \ldots,\left\{V_{i}\right\}\right\}$ is a finite non-empty set of vertices in $\mathbb{R}^{n}, i=0,1,2, \ldots, n$ and each vertex consistes of k-simplex graph, i.e. $\left\{V_{i}\right\}=\left\{\left\{v_{i 0}, e_{i 0}\right\},\left\{v_{i 1}, e_{i 1}\right\}, \ldots,\left\{v_{i k}, v_{i k}\right\}\right\}$ and $E(G)$ is 
a set of unordered pairs of distinct elements of $V(G)$.

2.2 The graph of 0-simplex vertices

It is represented as a simple graph, see Fig.(1).

It's adjacent and incidence are:

$$
A(G)=\left[\begin{array}{ll}
0 & 1_{\Delta^{0}} \\
1_{\Delta^{0}} & 0
\end{array}\right], \quad I(G)=\left[\begin{array}{l}
1_{\Delta^{0}} \\
1_{\Delta^{0}}
\end{array}\right]
$$

Where $\left(\Delta^{0}\right)$ refer to 0 -simplex graph.

\subsection{The graph of 1 -simplex vertices}

It has two types of dimensions of edges.

Type(1) The edge of 1-dimension see Fig.(2), Fig.(3), Fig.(4).

$V(G)=\left\{V_{0}=\left\{v_{01}, e_{01}, v_{02}\right\}, V_{1}=\left\{v_{11}, e_{11}, v_{12}\right\}\right\}, E(G)=\left\{e_{1}\right\}$.

Fig.(2)

The adjacent and incidence are:

$$
A(G)=\left[\begin{array}{ll}
0 & 1_{\Delta^{1}} \\
1_{\Delta^{1}} & 0
\end{array}\right]_{\overleftarrow{\uparrow}}, \quad I(G)=\left[\begin{array}{l}
1_{\Delta^{1}} \\
1_{\Delta^{1}}
\end{array}\right]_{\overleftarrow{1}}
$$

Where $\overleftarrow{1}$ refer to the dimension and form of the edge on the graph.

Fig.(3)

$$
A(G)=\left[\begin{array}{ll}
0 & 1_{\Delta^{1}} \\
1_{\Delta^{1}} & 0
\end{array}\right]_{1}^{\searrow}, \quad I(G)=\left[\begin{array}{l}
1_{\Delta^{1}} \\
1_{\Delta^{1}}
\end{array}\right]_{1}^{\searrow}
$$

Fig.(4)

$$
A(G)=\left[\begin{array}{ll}
0 & 1_{\Delta^{1}} \\
1_{\Delta^{1}} & 0
\end{array}\right]_{1 \leftarrow}, \quad I(G)=\left[\begin{array}{l}
1_{\Delta^{1}} \\
1_{\Delta^{1}}
\end{array}\right]_{1 \leftarrow}
$$

Type(2) The edge of 2-dimension see Fig.(5), Fig.(6).

Fig.(5)

The adjacent and incidence are:

$$
A(G)=\left[\begin{array}{ll}
0 & 1_{\Delta^{1}} \\
1_{\Delta^{1}} & 0
\end{array}\right]_{\overleftarrow{\Sigma}}, \quad I(G)=\left[\begin{array}{l}
1_{\Delta^{1}} \\
1_{\Delta^{1}}
\end{array}\right]_{\overleftarrow{\Sigma}}
$$

Fig.(6)

$$
A(G)=\left[\begin{array}{ll}
0 & 1_{\Delta^{1}} \\
1_{\Delta^{1}} & 0
\end{array}\right]_{\llcorner}, \quad I(G)=\left[\begin{array}{l}
1_{\Delta^{1}} \\
1_{\Delta^{1}}
\end{array}\right]_{2}
$$

\subsection{The graph of 2-simplex vertices}

In this case the edge will be in three types of dimensions.

Type(1) The edge of 1-dimension see Fig.(7), Fig.(8), Fig.(9), Fig.(10).

Fig.(7)

The adjacent and incidence will be in the form:

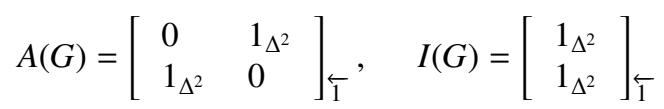


Fig.(8) where

$$
A(G)=\left[\begin{array}{ll}
0 & 1_{\Delta^{2}} \\
1_{\Delta^{2}} & 0
\end{array}\right]_{1 \leftarrow}, \quad I(G)=\left[\begin{array}{l}
1_{\Delta^{2}} \\
1_{\Delta^{2}}
\end{array}\right]_{1 \leftarrow}
$$

Fig.(9)

$$
A(G)=\left[\begin{array}{ll}
0 & 1_{\Delta^{2}} \\
1_{\Delta^{2}} & 0
\end{array}\right]_{1}, \quad I(G)=\left[\begin{array}{l}
1_{\Delta^{2}} \\
1_{\Delta^{2}}
\end{array}\right]_{1}
$$

Fig.(10)

$$
A(G)=\left[\begin{array}{ll}
0 & 1_{\Delta^{2}} \\
1_{\Delta^{2}} & 0
\end{array}\right]_{1 \longleftrightarrow}, \quad I(G)=\left[\begin{array}{l}
1_{\Delta^{2}} \\
1_{\Delta^{2}}
\end{array}\right]_{1 \longleftrightarrow}
$$

Where $(1 \longleftrightarrow)$ refer to the edge which connecte between the area of each simplex .

Type(2) The edge of 2-dimension see Fig.(11), Fig.(12), Fig.(13), Fig.(14).

Fig.(11)

It's adjacent and incidence are:

$$
A(G)=\left[\begin{array}{ll}
0 & 1_{\Delta^{2}} \\
1_{\Delta^{2}} & 0
\end{array}\right]_{\overleftarrow{\Sigma}}, \quad I(G)=\left[\begin{array}{l}
1_{\Delta^{2}} \\
1_{\Delta^{2}}
\end{array}\right]_{\overleftarrow{2}}
$$

Fig.(12)

$$
A(G)=\left[\begin{array}{ll}
0 & 1_{\Delta^{2}} \\
1_{\Delta^{2}} & 0
\end{array}\right]_{2}, \quad I(G)=\left[\begin{array}{l}
1_{\Delta^{2}} \\
1_{\Delta^{2}}
\end{array}\right]_{2}
$$

Fig.(13)

$$
A(G)=\left[\begin{array}{ll}
0 & 1_{\Delta^{2}} \\
1_{\Delta^{2}} & 0
\end{array}\right]_{\overleftarrow{2}}, \quad I(G)=\left[\begin{array}{l}
1_{\Delta^{2}} \\
1_{\Delta^{2}}
\end{array}\right]_{\overleftarrow{2}}
$$

Fig.(14)

$$
A(G)=\left[\begin{array}{ll}
0 & 1_{\Delta^{2}} \\
1_{\Delta^{2}} & 0
\end{array}\right]_{\swarrow}, \quad I(G)=\left[\begin{array}{l}
1_{\Delta^{2}} \\
1_{\Delta^{2}}
\end{array}\right]_{2}
$$

Type(3) The edge of 3-dimension see Fig.(15), Fig.(16).

Fig.(15)

The adjacent and incidence will be in the form:

$$
A(G)=\left[\begin{array}{ll}
0 & 1_{\Delta^{2}} \\
1_{\Delta^{2}} & 0
\end{array}\right]_{\overleftarrow{3}}, \quad I(G)=\left[\begin{array}{l}
1_{\Delta^{2}} \\
1_{\Delta^{2}}
\end{array}\right]_{\overleftarrow{3}}
$$

Fig.(16)

$$
A(G)=\left[\begin{array}{ll}
0 & 1_{\Delta^{2}} \\
1_{\Delta^{2}} & 0
\end{array}\right]_{\breve{3}}, \quad I(G)=\left[\begin{array}{l}
1_{\Delta^{2}} \\
1_{\Delta^{2}}
\end{array}\right]_{3}
$$

\subsection{The graph of simplex vertices in higher dimension}

The graph of n-simplex vertices has types of edges of higher dimensions.

We can represent these types of edges by matrices as the following: 
The $1^{\text {st }}$ type: $A(G)=\left[\begin{array}{ll}0 & 1_{\Delta^{n}} \\ 1_{\Delta^{n}} & 0\end{array}\right]_{n+1}, \quad I(G)=\left[\begin{array}{l}1_{\Delta^{n}} \\ 1_{\Delta^{n}}\end{array}\right]_{n+1}$

The $2^{\text {ed }}$ type: $A(G)=\left[\begin{array}{ll}0 & 1_{\Delta^{n}} \\ 1_{\Delta^{n}} & 0\end{array}\right]_{n}, \quad I(G)=\left[\begin{array}{l}1_{\Delta^{n}} \\ 1_{\Delta^{n}}\end{array}\right]_{n}$

The $3^{\text {ed }}$ type: $A(G)=\left[\begin{array}{ll}0 & 1_{\Delta^{n}} \\ 1_{\Delta^{n}} & 0\end{array}\right]_{n-1}, \quad I(G)=\left[\begin{array}{l}1_{\Delta^{n}} \\ 1_{\Delta^{n}}\end{array}\right]_{n-1}$

The $(n+1)$ type: $A(G)=\left[\begin{array}{ll}0 & 1_{\Delta^{n}} \\ 1_{\Delta^{n}} & 0\end{array}\right]_{1}, \quad I(G)=\left[\begin{array}{l}1_{\Delta^{n}} \\ 1_{\Delta^{n}}\end{array}\right]_{1}$

Lemma 1 Any graph $G$ of $n$-simplex vertices can be connected by edges of $(n+1, n, n-1, \ldots, 1)$ dimension.

Lemma 2 The edge which connect between two vertices of simplex graph has dimension equal to or less than one the largest number of 0 -faces that belong to any of the simplex graph.

\section{Folding of Geometric Graph of Simplex Vertices}

We can make many types of foldings.

Type(1) Folding of External Vertices and External Edges.

Fig.(17)

such that

$$
\begin{gathered}
A(G)=\left[\begin{array}{lll}
0 & 1_{\Delta^{1}} & 0 \\
1_{\Delta^{1}} & 0 & 1_{\Delta^{1}} \\
0 & 1_{\Delta^{1}} & 0
\end{array}\right] \stackrel{F_{1}}{\longrightarrow}\left[\begin{array}{ll}
0 & 1_{\Delta^{1}} \\
1_{\Delta^{1}} & 0
\end{array}\right] \stackrel{F_{2}}{\longrightarrow}\left[1_{\Delta^{1}}^{1}\right] \\
I(G)=\left[\begin{array}{ll}
1_{\Delta^{1}} & 0 \\
1_{\Delta^{1}} & 1_{\Delta^{1}} \\
0 & 1_{\Delta^{1}}
\end{array}\right] \stackrel{F_{1}}{\longrightarrow}\left[\begin{array}{l}
1_{\Delta^{1}} \\
1_{\Delta^{1}}
\end{array}\right] \stackrel{F_{2}}{\longrightarrow}[0]
\end{gathered}
$$

Where $1^{1}$ the upper suffix refers to the existence of loops.

Fig.(18)

where

$$
\begin{gathered}
A(G)=\left[\begin{array}{lll}
0 & 1_{\Delta^{1}} & 0 \\
1_{\Delta^{1}} & 0 & 1 \\
0 & 1_{\Delta^{1}} & 0
\end{array}\right] \stackrel{F_{1}}{\longrightarrow}\left[\begin{array}{ll}
0 & 1_{\Delta^{1}} \\
1_{\Delta^{1}} & 0
\end{array}\right] \stackrel{F_{2}}{\longrightarrow}\left[1_{\Delta^{1}}^{11}\right] \\
I(G)=\left[\begin{array}{ll}
1_{\Delta^{1}} & 0 \\
1_{\Delta^{1}} & 1_{\Delta^{1}} \\
0 & 1_{\Delta^{1}}
\end{array}\right] \stackrel{F_{1}}{\longrightarrow}\left[\begin{array}{l}
1_{\Delta^{1}} \\
1_{\Delta^{1}}
\end{array}\right] \stackrel{F_{2}}{\longrightarrow}[0]
\end{gathered}
$$

Fig.(19)

where

$$
\begin{gathered}
A(G)=\left[\begin{array}{lll}
0 & 1_{\Delta^{1}} & 0 \\
1_{\Delta^{1}} & 0 & 1_{\Delta^{1}} \\
0 & 1_{\Delta^{1}} & 0
\end{array}\right] \stackrel{F_{1}}{\longrightarrow}\left[\begin{array}{ll}
0 & 1_{\Delta^{1}} \\
1_{\Delta^{1}} & 0
\end{array}\right] \stackrel{F_{2}}{\longrightarrow}\left[1_{\Delta^{1}}^{(1)_{2}}\right] \\
I(G)=\left[\begin{array}{ll}
1_{\Delta^{1}} & 0 \\
1_{\Delta^{1}} & 1_{\Delta^{1}} \\
0 & 1_{\Delta^{1}}
\end{array}\right] \stackrel{F_{1}}{\longrightarrow}\left[\begin{array}{l}
1_{\Delta^{1}} \\
1_{\Delta^{1}}
\end{array}\right] \stackrel{F_{2}}{\longrightarrow}[0]
\end{gathered}
$$


Where $1^{(1)_{2}}$ refer to the loop of dimension 2.

Fig.(20)

$$
\begin{gathered}
A(G)=\left[\begin{array}{lll}
0 & 1_{\Delta^{2}} & 0 \\
1_{\Delta^{2}} & 0 & 1_{\Delta^{2}} \\
0 & 1_{\Delta^{2}} & 0
\end{array}\right] \stackrel{F_{1}}{\longrightarrow}\left[\begin{array}{ll}
0 & 1_{\Delta^{2}} \\
1_{\Delta^{2}} & 0
\end{array}\right] \stackrel{F_{2}}{\longrightarrow}\left[1_{\Delta^{2}}^{1}\right] \\
I(G)=\left[\begin{array}{ll}
1_{\Delta^{1}} & 0 \\
1_{\Delta^{1}} & 1_{\Delta^{1}} \\
0 & 1_{\Delta^{1}}
\end{array}\right] \stackrel{F_{1}}{\longrightarrow}\left[\begin{array}{l}
1_{\Delta^{1}} \\
1_{\Delta^{1}}
\end{array}\right] \stackrel{F_{2}}{\longrightarrow}[0]
\end{gathered}
$$

Fig.(21)

$$
\begin{gathered}
A(G)=\left[\begin{array}{lll}
0 & 1_{\Delta^{2}} & 0 \\
1_{\Delta^{2}} & 0 & 1_{\Delta^{2}} \\
0 & 1_{\Delta^{2}} & 0
\end{array}\right] \stackrel{F_{1}}{\longrightarrow}\left[\begin{array}{ll}
0 & 1_{\Delta^{2}} \\
1_{\Delta^{2}} & 0
\end{array}\right] \stackrel{F_{2}}{\longrightarrow}\left[1_{\Delta^{2}}^{11}\right] \\
I(G)=\left[\begin{array}{ll}
1_{\Delta^{2}} & 0 \\
1_{\Delta^{2}} & 1_{\Delta^{2}} \\
0 & 1_{\Delta^{2}}
\end{array}\right] \stackrel{F_{1}}{\longrightarrow}\left[\begin{array}{l}
1_{\Delta^{1}} \\
1_{\Delta^{1}}
\end{array}\right] \stackrel{F_{2}}{\longrightarrow}[0]
\end{gathered}
$$

Fig.(22)

$$
\begin{gathered}
A(G)=\left[\begin{array}{lll}
0 & 1_{\Delta^{2}} & 0 \\
1_{\Delta^{2}} & 0 & 1_{\Delta^{2}} \\
0 & 1_{\Delta^{2}} & 0
\end{array}\right]_{2} \stackrel{F_{1}}{\longrightarrow}\left[\begin{array}{ll}
0 & 1_{\Delta^{2}} \\
1_{\Delta^{2}} & 0
\end{array}\right]_{2} \stackrel{F_{2}}{\longrightarrow}\left[1_{\Delta^{2}}^{(1)_{2}}\right]_{2} \\
I(G)=\left[\begin{array}{ll}
1_{\Delta^{2}} & 0 \\
1_{\Delta^{2}} & 1_{\Delta^{2}} \\
0 & 1_{\Delta^{2}}
\end{array}\right]_{2} \stackrel{F_{1}}{\longrightarrow}\left[\begin{array}{l}
1_{\Delta^{1}} \\
1_{\Delta^{1}}
\end{array}\right]_{2} \stackrel{F_{2}}{\longrightarrow}[0]
\end{gathered}
$$

Fig.(23)

$$
\begin{gathered}
A(G)=\left[\begin{array}{lll}
0 & 1_{\Delta^{2}} & 0 \\
1_{\Delta^{2}} & 0 & 1 \\
0 & 1_{\Delta^{2}} & 0
\end{array}\right]_{3} \stackrel{F_{1}}{\longrightarrow}\left[\begin{array}{ll}
0 & 1_{\Delta^{2}} \\
1_{\Delta^{2}} & 0
\end{array}\right]_{3} \stackrel{F_{2}}{\longrightarrow}\left[1_{\Delta^{2}}^{(1)_{3}}\right]_{3} \\
I(G)=\left[\begin{array}{ll}
1_{\Delta^{2}} & 0 \\
1_{\Delta^{2}} & 1_{\Delta^{2}} \\
0 & 1_{\Delta^{2}}
\end{array}\right]_{3} \stackrel{F_{1}}{\longrightarrow}\left[\begin{array}{l}
1_{\Delta^{1}} \\
1_{\Delta^{1}}
\end{array}\right]_{3} \stackrel{F_{2}}{\longrightarrow}[0]
\end{gathered}
$$

Some loopes will take different shape see Fig.(24).

The adjacent and incidence will be in the form:

$$
\begin{gathered}
A(G)=\left[\begin{array}{lll}
0 & 1_{\Delta^{1}} & 0 \\
1_{\Delta^{1}} & 0 & 1_{\Delta^{1}} \\
0 & 1_{\Delta^{1}} & 0
\end{array}\right]_{2} \stackrel{F_{1}}{\longrightarrow}\left[\begin{array}{ll}
0 & 1_{\Delta^{1}} \\
1_{\Delta^{1}} & 0
\end{array}\right]_{2} \stackrel{F_{2}}{\longrightarrow}\left[1_{\Delta^{1}}^{(1) \overbrace{2}}\right]_{2} \\
I(G)=\left[\begin{array}{ll}
1_{\Delta^{2}} & 0 \\
1_{\Delta^{2}} & 1_{\Delta^{2}} \\
0 & 1_{\Delta^{2}}
\end{array}\right]_{2} \stackrel{F_{1}}{\longrightarrow}\left[\begin{array}{l}
1_{\Delta^{1}} \\
1_{\Delta^{1}}
\end{array}\right]_{2} \stackrel{F_{2}}{\longrightarrow}[0]
\end{gathered}
$$

Type(2) Folding of internal edges and internal vertices see Fig.(25).

Where

$$
A(G)=\left[\begin{array}{lll}
0 & 1_{\Delta^{1}} & 0 \\
1_{\Delta^{1}} & 0 & 1_{\Delta^{1}} \\
0 & 1_{\Delta^{1}} & 0
\end{array}\right] \stackrel{F_{1}}{\longrightarrow}\left[\begin{array}{lll}
1_{\Delta^{0}}^{1} & 1_{\Delta^{0}} & 0 \\
1_{\Delta^{0}} & 1_{\Delta^{0}}^{1} & 1_{\Delta^{0}} \\
0 & 1_{\Delta^{0}} & 1_{\Delta^{0}}^{1}
\end{array}\right] \stackrel{F_{2}}{\longrightarrow}\left[\begin{array}{ll}
1_{\Delta^{0}}^{11} & 1_{\Delta^{0}} \\
1_{\Delta^{0}} & 1_{\Delta^{0}}^{1}
\end{array}\right] \stackrel{F_{3}}{\longrightarrow}\left[1_{\Delta^{0}}^{1111}\right]
$$




$$
I(G)=\left[\begin{array}{ll}
1_{\Delta_{1}^{1}} & 0 \\
1_{\Delta_{1}^{1}} & 1_{\Delta_{1}^{1}} \\
0 & 1_{\Delta_{1}^{1}}
\end{array}\right] \stackrel{F_{1}}{\longrightarrow}\left[\begin{array}{ll}
1_{\Delta_{0}^{0}} & 0 \\
1_{\Delta_{0}^{0}} & 1_{\Delta_{0}^{0}} \\
0 & 1_{\Delta_{0}^{0}}
\end{array}\right] \stackrel{F_{2}}{\longrightarrow}\left[\begin{array}{l}
1_{\Delta_{0}^{0}} \\
1_{\Delta_{0}^{0}}
\end{array}\right] \stackrel{F_{3}}{\longrightarrow}[0]
$$

Where $\left(\Delta_{1}\right)$ refer to number of internal edges.

Fig.(26)

$$
\begin{aligned}
& A(G)=\left[\begin{array}{lll}
0 & 1_{\Delta^{1}} & 0 \\
1_{\Delta^{1}} & 0 & 1_{\Delta^{1}} \\
0 & 1_{\Delta^{1}} & 0
\end{array}\right]_{2} \stackrel{F_{1}}{\longrightarrow}\left[\begin{array}{lll}
1_{\Delta^{0}}^{(1)_{2}} & 1_{\Delta^{0}} & 0 \\
1_{\Delta^{0}} & 1_{\Delta^{0}}^{(1)_{2}} & 1_{\Delta^{0}} \\
0 & 1_{\Delta^{0}} & 1_{\Delta^{0}}^{(1)_{2}}
\end{array}\right]_{2} \stackrel{F_{2}}{\longrightarrow}\left[\begin{array}{ll}
1_{\Delta^{0}}^{(11)_{2}} & 1_{\Delta^{0}} \\
1_{\Delta^{0}} & 1_{\Delta^{0}}^{(1)_{2}}
\end{array}\right]_{2} \stackrel{F_{3}}{\longrightarrow}\left[1_{\Delta^{0}}^{(111)_{2}(1)_{3}}\right]_{2}
\end{aligned}
$$

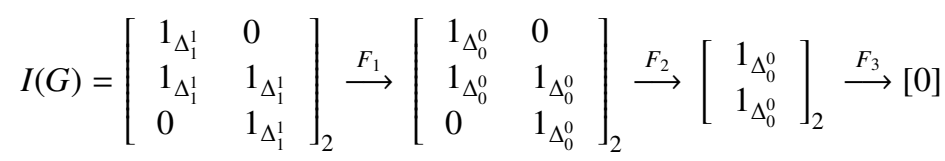

Fig.(27)

$$
\begin{aligned}
& A(G)=\left[\begin{array}{lll}
0 & 1_{\Delta^{2}} & 0 \\
1_{\Delta^{2}} & 0 & 1_{\Delta^{2}} \\
0 & 1_{\Delta^{2}} & 0
\end{array}\right] \stackrel{F_{1}}{\longrightarrow}\left[\begin{array}{lll}
1_{\Delta^{1}}^{1} & 1_{\Delta^{1}} & 0 \\
1_{\Delta^{1}} & 1_{\Delta^{1}}^{1} & 1_{\Delta^{1}} \\
0 & 1_{\Delta^{1}} & 1_{\Delta^{1}}^{1}
\end{array}\right] \stackrel{F_{2}}{\longrightarrow}\left[\begin{array}{lll}
1_{\Delta^{0}}^{11} & 1_{\Delta^{0}} & 0 \\
1_{\Delta^{0}} & 1_{\Delta^{0}}^{11} & 1_{\Delta^{0}} \\
0 & 1_{\Delta^{0}} & 1_{\Delta^{0}}^{11}
\end{array}\right] \stackrel{F_{3}}{\longrightarrow}\left[\begin{array}{ll}
1_{\Delta^{0}}^{1111} & 1_{\Delta^{0}} \\
1_{\Delta^{0}} & 1_{\Delta^{0}}^{11}
\end{array}\right] \stackrel{F_{4}}{\longrightarrow}\left[1_{\Delta^{0}}^{111111}\right]
\end{aligned}
$$

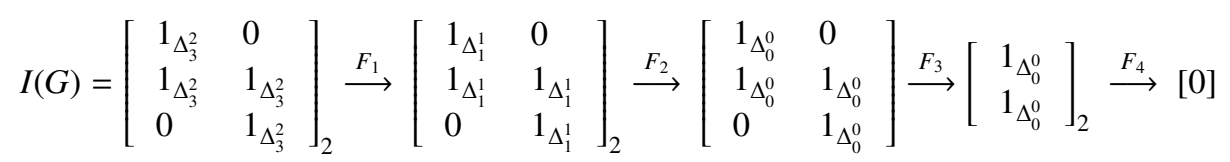

Fig.(28)

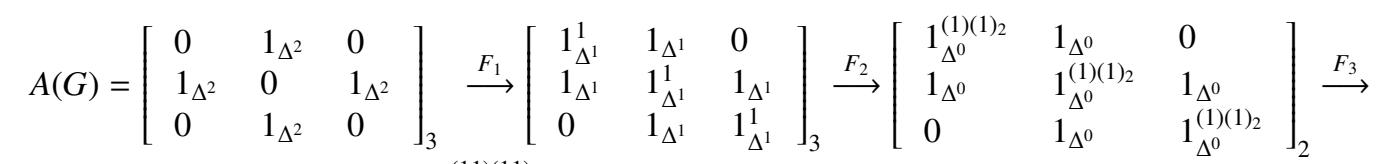

$$
\begin{aligned}
& {\left[\begin{array}{ll}
1_{\Delta^{0}}^{(11)(11)_{2}} & 1_{\Delta^{0}} \\
1_{\Delta^{0}} & 1_{\Delta^{0}}^{(1)(1)_{2}}
\end{array}\right]_{2} \stackrel{F_{4}}{\longrightarrow}\left[1_{\Delta^{0}}^{(111)(11)_{2}(1)_{3}}\right]}
\end{aligned}
$$

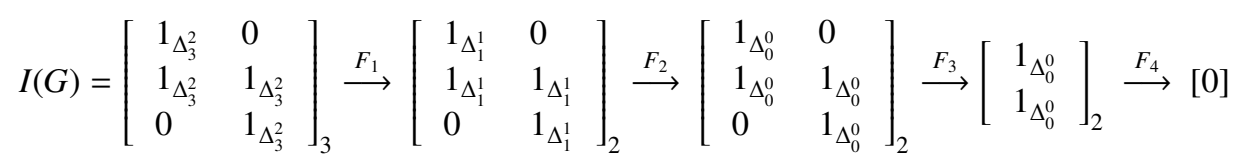

Type(3) In the next graph we will fold the length of the edges on each other see Fig.(29), Fig(30).

Fig.(29)

$$
\begin{gathered}
A(G)=\left[\begin{array}{lll}
0 & 1_{\Delta^{1}} & 0 \\
1_{\Delta^{1}} & 0 & 1_{\Delta^{1}} \\
0 & 1_{\Delta^{1}} & 0
\end{array}\right] \stackrel{F_{1}}{\longrightarrow}\left[\begin{array}{lll}
0 & 1_{\Delta^{1}} & 0 \\
1_{\Delta^{1}} & 0 & 1_{\Delta^{1}} \\
0 & 1_{\Delta^{1}} & 0
\end{array}\right] \stackrel{F_{2}}{\longrightarrow}\left[\begin{array}{ll}
0 & 1_{\Delta^{1}} \\
1_{\Delta^{1}} & 0
\end{array}\right] \stackrel{F_{3}}{\longrightarrow}\left[0_{\Delta^{1}}\right] \\
I(G)=\left[\begin{array}{ll}
1_{\Delta^{1}} & 0 \\
1_{\Delta^{1}} & 1_{\Delta^{1}} \\
0 & 1_{\Delta^{1}}
\end{array}\right] \stackrel{F_{1}}{\longrightarrow}\left[\begin{array}{ll}
1_{\Delta^{1}} & 0 \\
1_{\Delta^{1}} & 1_{\Delta^{1}} \\
0 & 1_{\Delta^{1}}
\end{array}\right] \stackrel{F_{2}}{\longrightarrow}\left[\begin{array}{l}
1_{\Delta^{1}} \\
1_{\Delta^{1}}
\end{array}\right] \stackrel{F_{3}}{\longrightarrow}[0]
\end{gathered}
$$

Fig.(30)

$$
\begin{gathered}
A(G)=\left[\begin{array}{lll}
0 & 1_{\Delta^{2}} & 0 \\
1_{\Delta^{2}} & 0 & 1 \\
0 & 1_{\Delta^{2}} & 0
\end{array}\right]_{2} \stackrel{F_{1}}{\longrightarrow}\left[\begin{array}{lll}
0 & 1_{\Delta^{2}} & 0 \\
1_{\Delta^{2}} & 0 & 1_{\Delta^{2}} \\
0 & 1_{\Delta^{2}} & 0
\end{array}\right]_{2} \stackrel{F_{2}}{\longrightarrow}\left[\begin{array}{ll}
0 & 1_{\Delta^{2}} \\
1_{\Delta^{2}} & 0
\end{array}\right]_{2} \stackrel{F_{3}}{\longrightarrow}\left[0_{\Delta^{2}}\right] \\
I(G)=\left[\begin{array}{ll}
1_{\Delta^{2}} & 0 \\
1_{\Delta^{2}} & 1_{\Delta^{2}} \\
0 & 1_{\Delta^{2}}
\end{array}\right]_{2} \stackrel{F_{1}}{\longrightarrow}\left[\begin{array}{ll}
1_{\Delta^{2}} & 0 \\
1_{\Delta^{2}} & 1_{\Delta^{2}} \\
0 & 1_{\Delta^{2}}
\end{array}\right]_{2} \stackrel{F_{2}}{\longrightarrow}\left[\begin{array}{l}
1_{\Delta^{2}} \\
1_{\Delta^{2}}
\end{array}\right]_{2} \stackrel{F_{3}}{\longrightarrow}[0]
\end{gathered}
$$


Type(4) The folding which reduce the volume of the graph, the incidence and adjacent matrices are of the same type see Fig.(31).

$$
\begin{aligned}
& A(G)=\left[\begin{array}{lll}
0 & 1_{\Delta^{1}} & 0 \\
1_{\Delta^{1}} & 0 & 1_{\Delta^{1}} \\
0 & 1_{\Delta^{1}} & 0
\end{array}\right] \stackrel{F_{1}}{\longrightarrow}\left[\begin{array}{lll}
0 & 1_{\Delta^{1}} & 0 \\
1_{\Delta^{1}} & 0 & 1_{\Delta^{1}} \\
0 & 1_{\Delta^{1}} & 0
\end{array}\right] \stackrel{F_{2}}{\longrightarrow}\left[\begin{array}{lll}
0 & 1_{\Delta^{1}} & 0 \\
1_{\Delta^{1}} & 0 & 1_{\Delta^{1}} \\
0 & 1_{\Delta^{1}} & 0
\end{array}\right] \underset{n \rightarrow \infty}{\longrightarrow} \underset{n \rightarrow m}{\operatorname{Lim}} F_{n}[0]
\end{aligned}
$$

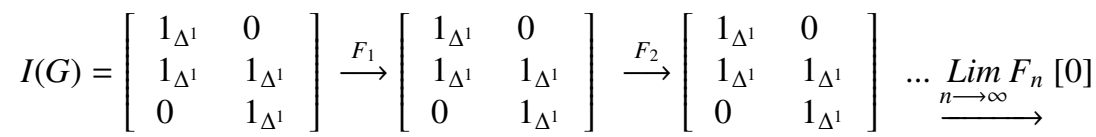

Theorem The end of the limit of foldings of simplex graph of dimension $n$ is the 0-simplex graph.

Proof: Let $G$ is a simplex graph of dimension $n, f$ is a folding. $f_{1:} G \longrightarrow G$ such that $f_{1}\left(E_{1}\right)=E_{2}$ then $f_{1}(G)=G_{1}$, $\operatorname{dim} E_{1}=\operatorname{dim} E_{2}$. Let $f_{2}\left(G_{1}\right)=G_{2}, f_{2}\left(E_{2}\right)=E_{3}, \operatorname{dim} E_{2}=\operatorname{dim} E_{3}, \ldots, f_{n}\left(G_{n-1}\right)=G_{n}, f_{n}\left(E_{n}\right)=E_{n+1}, \underset{n \rightarrow \infty}{\lim f_{n}\left(G_{n-1}\right)=H, H}$ of $(n-1)$ dimension.

And $g_{1}: H \longrightarrow H$ such that $g_{1}\left(E_{1}\right)=E_{2}$ then $g_{1}(H)=H_{1}, \operatorname{dim} E_{1}=\operatorname{dim} E_{2}$. Let $g_{2}\left(H_{1}\right)=H_{2}, g_{2}\left(E_{2}\right)=E_{3}$, $\operatorname{dim} E_{2}=\operatorname{dim} E_{3}, \ldots, g_{n}\left(H_{n-1}\right)=H_{n}, g_{n}\left(E_{n}\right)=E_{n+1}, \lim _{n \rightarrow \infty} g_{n}\left(H_{n-1}\right)=L, L$ of $(n-2)$ dimension. And $\lim _{n \rightarrow \infty} \lim _{n}\left(G_{n}\right)=$ 0-simplex graph see Fig.(31).

\section{References}

El-Ghoul, M. Unfolding of graph and uncertain graph. The Australian Mathematics Journal, Sandy Bay 7006, Tasmania, Australia.

Gibbson, A. (1995). Algorithmic graph theory. Cambridge University Press, Cambridge, UK.

Giblin, P. J. (1977). Graphs, surfaces and homology: an introduction to algebraic topology. Chapman and Hall, Ltd, London.

Gross, J. L., \& Tucker, T. W. (1987). Topological graph theory. Jon Wiley \& Sons, Inc, Canada.

Hatcher, A. (2002). Algebraic topology. Cambridge University Press.

Wilson, R. J. (1972). Introduction to graph theory. Oliver and Boyd, Edinburgh.

Wilson, R. J., \& Watkins, J. J. (1990). Graphs: an introductory approach-a first course in discrete mathematics. Jon Wiley \& Sons, Inc, Canada.

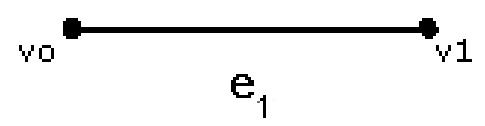

Figure(1)

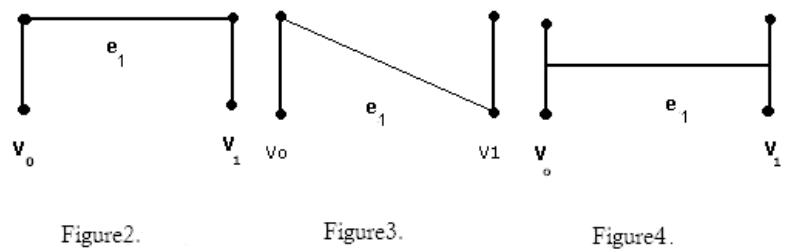

Figure 1-4 


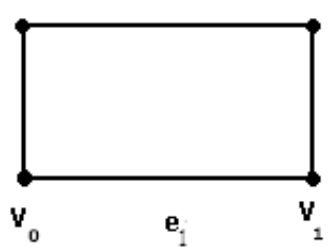

Figure5

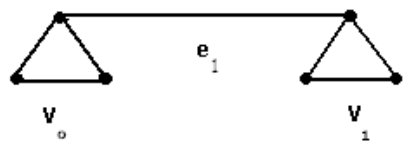

Figure7.

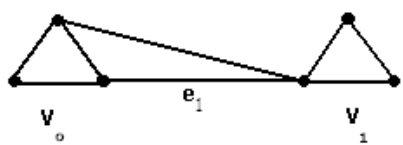

Figure12

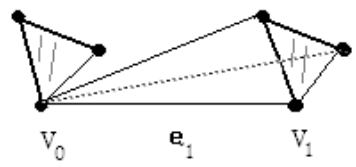

Figure14

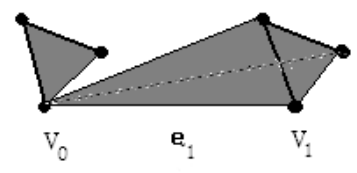

Figure15

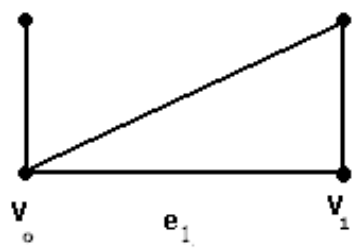

Figure6.

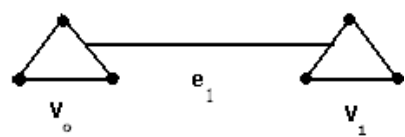

Figure8.

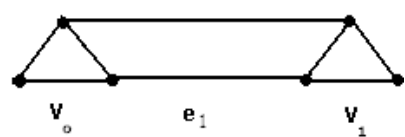

Figure11

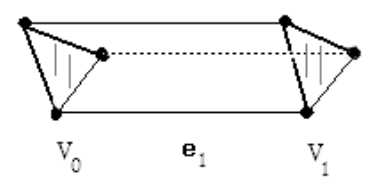

Figure13

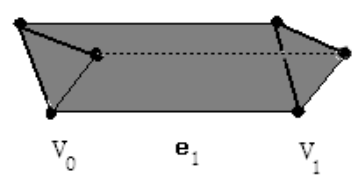

Figure16.

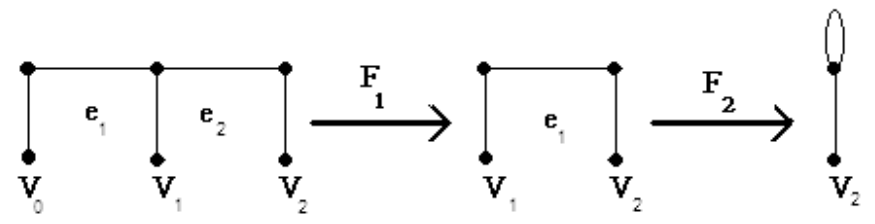

Figure 17.

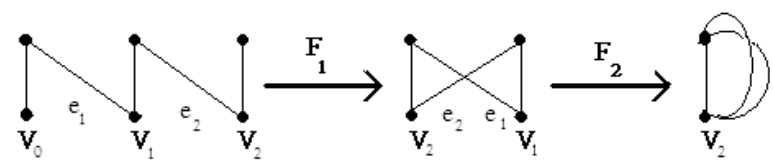

Figure18

Figure 5-18 


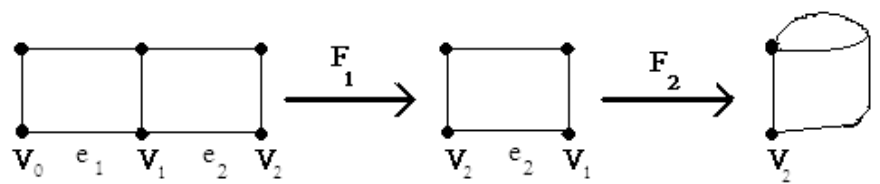

Figure19
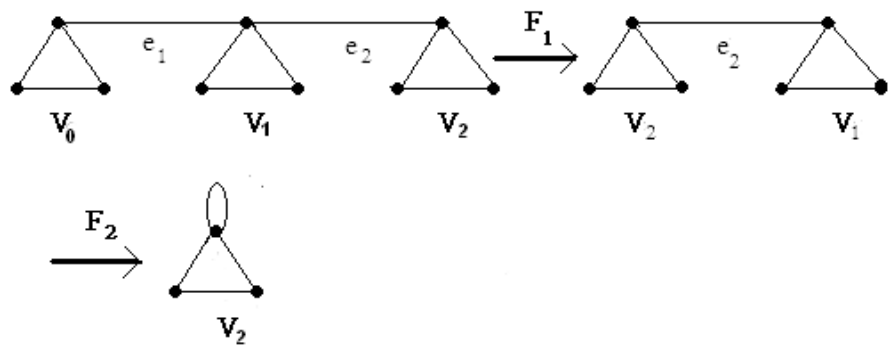

Figure 20
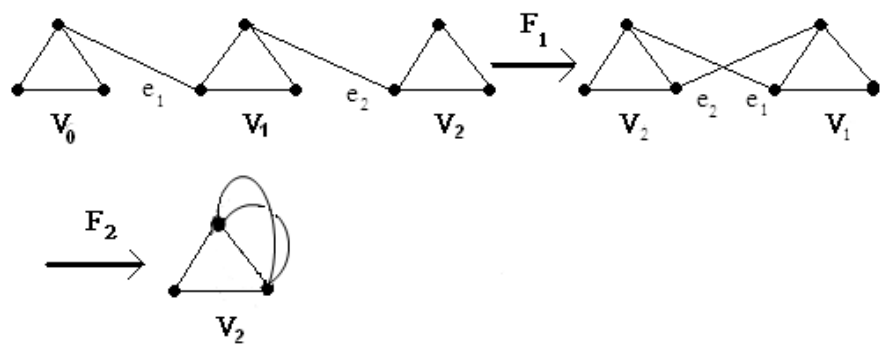

Figure 21
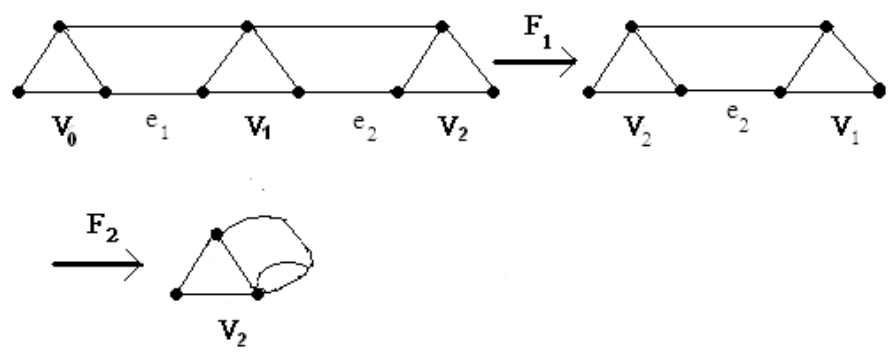

Figure22

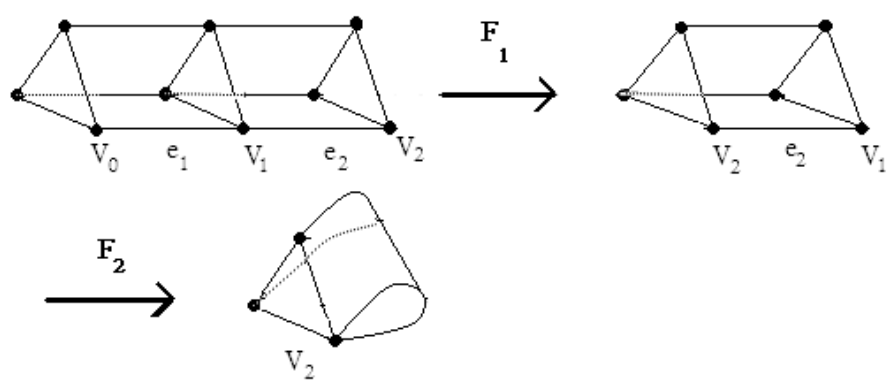

Figure23.

Figure 19-23 


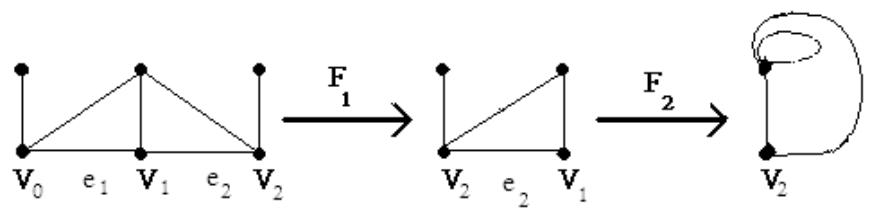

Figure24.
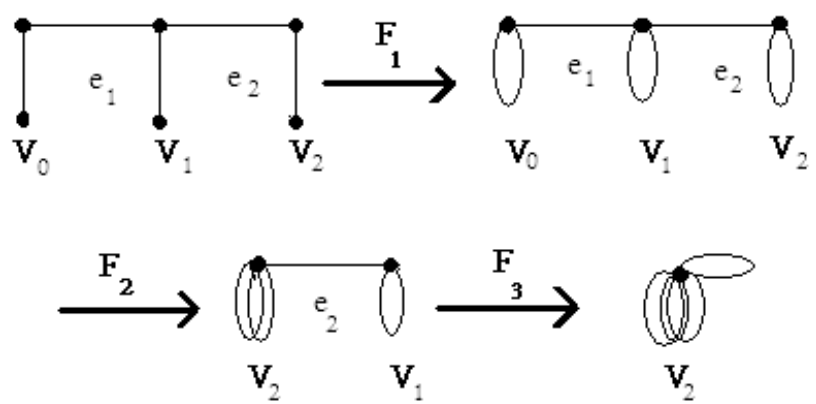

Figure25.
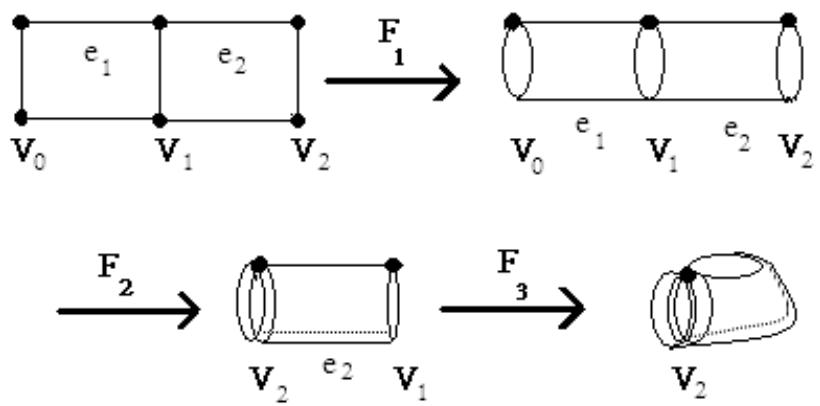

Figure26.
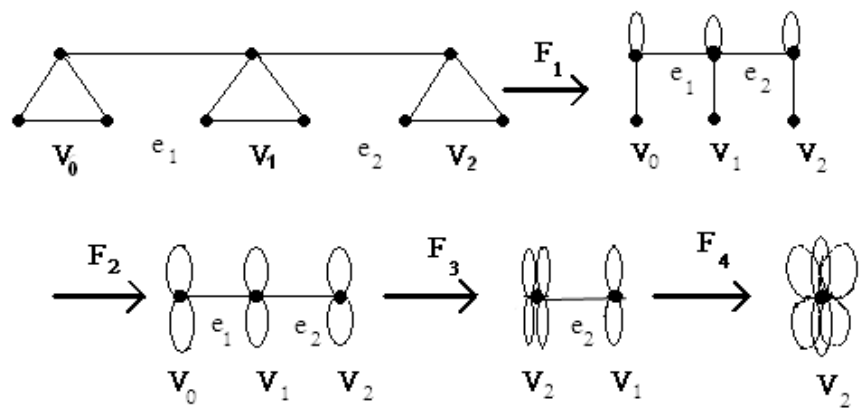

Figure27

Figure 24-27 

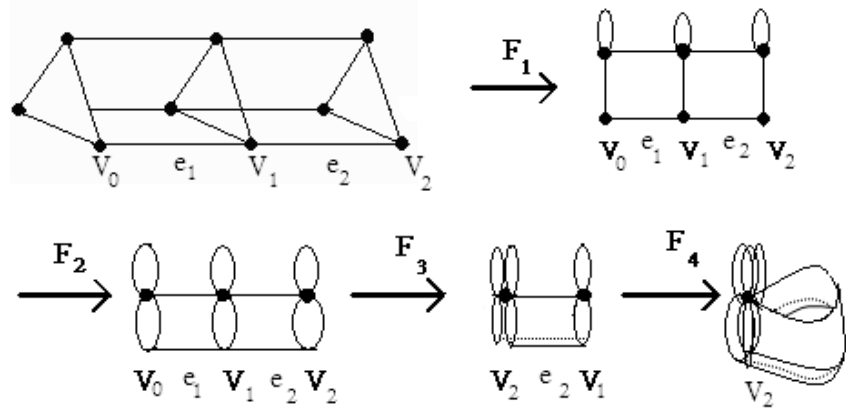

Figure28.

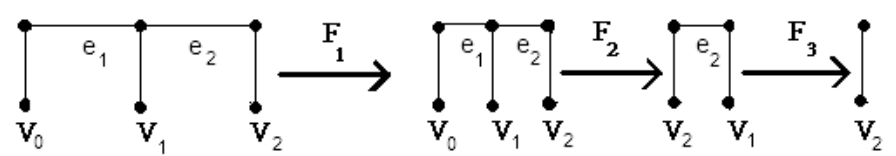

Figure29
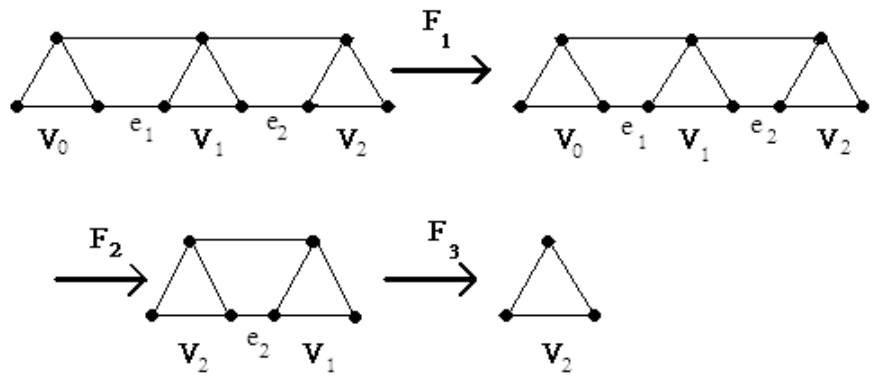

Figure 30.
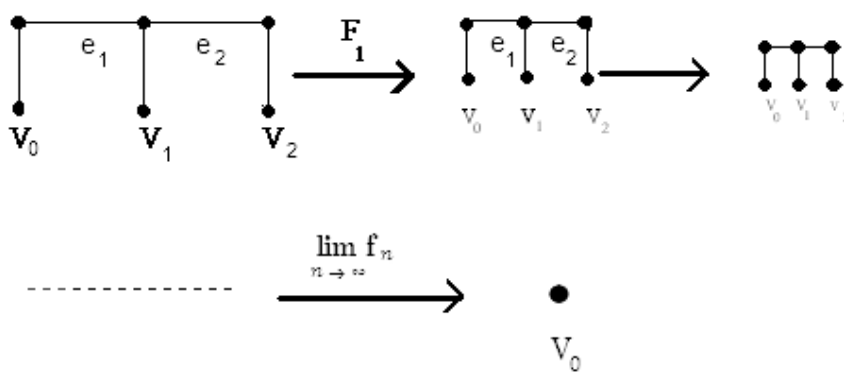

Figure31.

Figure 28-31 